\title{
Services immatériels et relationnels : intensité du travail et santé
}

Immaterial services: work intensity and health

\section{Christian du Tertre}

\section{(2) OpenEdition}

\section{Journals}

Édition électronique

URL : http://journals.openedition.org/activites/1567

DOI : 10.4000/activites. 1567

ISSN : 1765-2723

\section{Éditeur}

ARPACT - Association Recherches et Pratiques sur les ACTivités

\section{Référence électronique}

Christian du Tertre, "Services immatériels et relationnels : intensité du travail et santé », Activités [En ligne], 2-1 | avril 2005, mis en ligne le 02 avril 2005, consulté le 19 avril 2019. URL : http:// journals.openedition.org/activites/1567; DOI : 10.4000/activites.1567

\section{(9) $\odot \Theta \Theta$}

Activités est mis à disposition selon les termes de la licence Creative Commons Attribution - Pas d'Utilisation Commerciale - Pas de Modification 4.0 International. 


\title{
Services immatériels et relationnels : intensité du travail et santé \\ Christian du Tertre
}

Professeur des universités en sciences économiques Directeur d'ATEMIS

Université Paris-7-Denis Diderot - GERME chdutertre@aol.com

\begin{abstract}
Immaterial services : work intensity and health

Immaterial and relational services occupy an increasingly important place in economy and growth dynamics. This explains why private firms and public organizations are trying to rationalize them.

Based on a characterization of immaterial economy, this paper illustrates the importance of time for regulating (behavioural adjustments) and of time intended for immaterial investments pertaining to regulating modes of workload intensity (training, reporting, evaluation...) as well as their impact on health
\end{abstract}

\section{KEYWORDS}

Services, service relationship, immaterial service, work intensity, health.

La crise structurelle que traversent les économies des pays de l'OCDE depuis la fin des années soixante-dix, est marquée par une accélération de l'évolution de la structure des emplois au profit des activités de service. Parallèlement à ce constat, il faut prendre la mesure d'une autre transformation structurelle : l'importance prise par les « activités immatérielles » fondées sur une « relation de service $»^{1}$, tant dans les secteurs d'activité relevant des services que dans les secteurs industriels.

Dans la mesure où ces « relations de service » modifient le contenu du travail comme sa finalité, elles mobilisent de plus en plus l'attention des acteurs socio-économiques : les directions d'entreprises et d'organismes publics ou para-publics, d'un côté, dans leur volonté d'établir un lien avec la performance ou la modernisation de leur organisation; les salariés, de l'autre, dans leur volonté de se repérer et d'appréhender les enjeux nouveaux auxquels ils sont confrontés. C'est en particulier le cas dans le cadre de la santé.

La question se pose de savoir dans quelles conditions apparaissent de nouvelles pathologies associées à la montée en puissance des « relations de service » soumises à des contraintes temporelles de plus en plus serrées. Dans cette perspective, une double interrogation émerge : quelle problématique économique mobiliser pour analyser le rapport des «activités immatérielles » à l'intensité du travail ; quels liens établir, dans le cadre de ces activités, entre l'intensité du travail et la santé, plus particulièrement les TMS.

Après avoir analysé les principales caractéristiques des activités immatérielles et relationnelles qui ont une incidence sur le travail et son contrôle, cet article tente de préciser les nouvelles clés de lecture analytique nécessaires à l'approche de l'intensité du travail et les conséquences économiques et humaines des processus d'intensification. Suivant une tradition régulationiste ${ }^{2}$, l'article se propose

1. On entend par « activités immatérielles et relationnelles » les activités de travail dont les effets ne sont pas tangibles, dans le sens où ils ne sont pas mesurables; on entend par « relation de service » la relation directe entre un prestataire de service et un bénéficiaire. Cette relation peut s'établir entre des acteurs appartenant à une même organisation ou à des organisations différentes. 
de comprendre les principales corrélations qu'entretient l'intensité du travail avec la dynamique de création de la valeur, et d'identifier les conditions d'émergence de nouveaux dispositifs institutionnels, notamment à propos de la santé, associés aux enjeux que posent la subjectivité et l'intersubjectivité dans la production de ces services. Il soulignera, alors, l'importance d'une approche pluridisciplinaire tournée non seulement vers les sciences sociales, mais également vers les sciences de l'homme, notamment la psychologie du travail.

\section{1.- Les principales caractéristiques de « la configuration productive de services immatériels et relationnels »}

La plupart des économistes classent les activités productives à partir des nomenclatures de l'INSEE, en secteurs et sous-secteurs, c'est-à-dire sur la base d'un critère qui instruit le niveau ${ }^{3}$ d'homogé- $^{\prime}$ néité des produits ou des services mais qui fait abstraction des conditions de leur production, c'est-àdire de l'exercice du travail réel. De telle nomenclatures ne nous aident pas à appréhender les modifications du travail et l'ampleur de leurs impacts. Pour ce dégager de ces approches qui privilégient le rapport au marché vis-à-vis du travail, la problématique de cet article classera les activités sur la base du concept de «configuration productive ${ }^{4}$. Il s'agit d'appréhender les activités à partir des liens qui s'établissent, au sein du procès de production lui-même, entre les compétences des salariés - voire des bénéficiaires - les technologies et l'organisation. Ces activités s'opèrent dans des espaces traditionnellement intitulés «productifs » ou «fonctionnels », sous une contrainte de valorisation, c'est-à-dire d'accumulation du capital.

Dans cette perspective, il est possible d'établir une distinction entre huit configurations productives différentes dont la « configuration des services immatériels et relationnels ». Cette dernière configuration est d'autant plus importante à prendre en compte aujourd'hui, pour l'analyse économique et l'analyse en sciences sociales, qu'elle occupe une place centrale dans le nouveau régime d'accumulation ${ }^{5}$ en émergence depuis le milieu des années soixante-dix (du Tertre, 2002).

\section{1.- Un rôle croissant des activités immatérielles dans la dynamique économique}

D'un côté, les activités immatérielles et relationnelles structurent, de plus en plus, la consommation des ménages, de l'autre, l'investissement des entreprises. En effet, la consommation des ménages est marquée par un glissement progressif vers les services, et plus particulièrement ceux qui sont intensifs en « relation de service» : formation, santé, tourisme, culture, soins du corps, sports, ... Il faut noter, également, que la consommation de produits manufacturés s'accompagne souvent de services

2. La théorie de la régulation est une théorie économique institutionnaliste apparue en France au milieu des années soixante-dix qui accorde une place centrale aux transformations du « rapport salarial ». Dans cette école de pensée, un réseau de chercheurs s'est particulièrement engagé dans l'analyse des dimensions servicielle et immatérielle de la dynamique économique remettant en cause les «Régulations sectorielles et territoriales » issues des années soixante/ soixante-dix.

3. Selon le niveau d'agrégation utilisée, les nomenclatures se présentent, aujourd'hui, selon une base européenne, en quatre, seize ou trente-six secteurs (NEP 4, 16 ou 36). Dans le cadre des activités de service un second critère est utilisé : la nature du bénéficiaire (entreprises ou ménages).

La théorie de la régulation a développé une problématique de l'approche sectorielle plus complexe, en introduisant, d'une part, une dimension historique et institutionnelle, d'autre part, une dimension analytique des conditions sociales de production de la valeur (Boyer, 1990 ; du Tertre 1989, 2002). Les secteurs sont, ainsi, des construits historiques et institutionnels particuliers en cours de déstructuration / restructuration, compte-tenu, notamment, de la place nouvelle de l'immatériel dans les processus d'accumulation. (cf. travaux du réseau RST de l'association « Recherche et Régulation »). 
associés, reposant, eux mêmes, sur une « relation de service »; les entreprises industrielles vendant des « bouquets » produit-services (maintenance, services financiers, formation, conseil...). L'investissement des entreprises, de son côté, évolue en structure au profit des «investissements immatériels » par rapport aux investissements matériels que sont les machines-outils, les équipements informatiques, les bâtiments... On entend par investissements immatériels, les activités de recherche/développement et d'innovation, de communication et d'information, de professionnalisation des salariés, de conseil et d'expertise, ... Ces investissements reposent, eux aussi, sur une « relation de service » déployée en interne à l'entreprise ou en externe avec un prestataire de service. L'efficacité de l'investissement va dépendre en grande partie de la qualité de cette « relation de service ».

De manière concomitante, les organismes publics et para-publics ont besoin de faire évoluer leur organisation afin de répondre à une demande de plus en plus personnalisée. Il ne s'agit plus d'offrir simplement le même service pour tous, mais de répondre au droit pour tous à un service personnalisé. Dans ce cadre, l'attention au bénéficiaire est un levier de modernisation fondamental. Il faut noter cependant que cette évolution ne peut pas se produire sans attention aux coûts et aux équilibres budgétaires. La « relation de service » n'est pas sollicitée uniquement dans une perspective de changement qui place les usagers au cœur des processus, mais aussi dans son lien avec la notion de performance qui intègre une dimension financière.

La nouveauté d'ordre économique ne tient pas tant à l'existence de ces activités immatérielles, en elles-mêmes -elles existent de longue date- mais de leur rôle central dans la dynamique économique. L'aspect nouveau tient au fait que les directions d'entreprises et d'organismes publics vont chercher à les rationaliser. Les activités immatérielles et relationnelles apparaissent, alors, au centre de tensions, notamment autour de l'intensité du travail, mais aussi de la qualité des services, ayant des répercussions sur la santé des salariés. Mais pour saisir ces tensions, il nous faut, maintenant, analyser les caractéristiques de ces activités et les questions nouvelles que posent leur insertion dans des processus de valorisation économique.

4. Le concept de « configuration productive » élargit le concept marxien de « procès de travail » en étendant le champ de l'analyse à trois phénomènes :

- la création de valeur est liée à l'irruption du bénéficiaire du service au sein même des activités de travail (la

« coproduction » du service) ;

- la création de valeur est liée à la qualité des agencements entre acteurs n'appartenant pas à la même entreprise, voire simplement aux mêmes ateliers, chantiers, sites ou bureaux. Ce concept permet, aussi, de prendre en compte l'impact des externalités sur la création de valeur, les articulations entre les gains de productivité (économies internes) et les économies externes, perçues comme des ressources par l'entreprise ;

- la création de valeur est associée à l'impact de la qualité du processus productif et de son organisation sur le renouvellement et l'enrichissement des ressources, notamment dans le domaine des compétences.

On peut distinguer huit « configurations productives » (une associée à l'agriculture, pêche, ...; trois au domaine de l'industrie et de la construction, quatre au domaine des services) : la configuration du vivant ; la configuration manufacturière de masse ; la configuration de process ; la configuration de chantier ; la configuration logistique ; la configuration de réparation-maintenance ; la configuration de services informationnels ; la configuration de services immatériels et relationnels.

Dans la réalité, une entreprise est amenée à combiner plusieurs configurations différentes ; mais l'une joue toujours un rôle repère et moteur, structurant le rapport de l'entreprise aux déterminants de la productivité et des externalités, aux conditions de renouvellement des ressources ; c'est-à-dire aux conditions de réalisation de la valeur.

5. Dans la théorie de la régulation une distinction est opérée entre « régime d'accumulation » et « régime de développement » (Boyer, \& Saillard, 2002). Régime de développement = régime d'accumulation + mode de régulation. En d'autres termes, un même régime d'accumulation peut conduire à des « régimes de développement » différents selon les modes de régulation mis en place par les groupes sociaux, les organisations et les institutions. 


\section{2.- Les principales caractéristiques des « services immatériels et relationnels » et l'enjeu de la santé}

La « configuration productive de services immatériels et relationnels » est caractérisée par :

- la réalisation d'un service (on s'inscrit, ici, dans la lignée des travaux de J. Gadrey, 1992, 1996 ${ }^{6}$ ) dont les effets utiles ne sont pas tangibles au sens de non mesurables. Les propriétés transformées au cours du service ne relèvent pas de normes permettant la pratique de mesures, mais de dimensions immatérielles et subjectives relevant d'une évaluation ${ }^{7}$;

- les effets utiles de ces services ont deux types d'impacts : des impacts immédiats sur des bénéficiaires directs, mais aussi des impacts «médiats». Certains effets peuvent apparaître avec un décalage temporel (par exemple, les connaissances acquises prennent sens après plusieurs mois), et/ou se propager en "réseau », auprès d'acteurs non directement concernés par la prestation (le soin d'une grippe, les activités sociales de quartier). Les effets parfois dits « externes » peuvent être, ainsi, très importants ;

- la coproduction propre à toute activité de service s'opère en grande partie dans un face-à-face prestataire / bénéficiaire. L'irruption de l'usager, du client..., du bénéficiaire au sein même du procès de travail devient une caractéristique «clé » de ces activités. A la différence des activités manufacturières où le client est tenu en dehors du procès de travail, ou des activités de service « tangibles » où le bénéficiaire est souvent limité à une activité informationnelle à la marge du procès de travail ${ }^{8}$ dans le cas des activités immatérielles et relationnelles, le bénéficiaire est au cour du dispositif productif, sur un plan spatial et temporel ;

- la coproduction se déploie dans une relation directe entre les deux acteurs (ou systèmes d'acteurs) mais de manière asymétrique. Si les deux parties sont actives, l'une travaille dans le cadre du salariat ${ }^{9}$, l'autre développe une activité en tant que « client », « patient », « usager »... La réalité de cette asymétrie et leur mode de représentation auront une incidence forte sur la subjectivité de chaque type d'acteur, leur intersubjectivité, et va avoir un impact important sur la qualité des effets utiles du service comme sur les moyens mobilisés pour l'obtenir.

La «relation de service» et l'intersubjectivité qui lui est associée, vont se déployer dans cinq registres :

- l'informationnel. A côté des informations formalisées, s'échangent des informations non formalisées par l'intermédiaire du corps de chaque acteur mobilisant un ou plusieurs des cinq sens des êtres humains ;

- le contractuel. A côté de contrats formels, la relation engage des conventions, des systèmes de droits et de devoirs tacites, contribuant à l'instauration d'un climat de confiance ;

- le social. La compréhension de l'autre s'opère à travers la représentation sociale de chaque acteur ;

- le technique et professionnel. Le bénéficiaire est amené à mobiliser des compétences qui entrent en résonance avec celles du prestataire, afin de coopérer avec ce dernier ;

- le psychique. L'activité se réalise sous le regard de l'autre, dans le dialogue à l'autre, engageant une activité de transfert et de contre-transfert.

6. Un prestataire de service transforme les propriétés d'un support (du service) qui n'appartient pas au prestataire mais au bénéficiaire. Ce support peut être le bénéficiaire lui-même (entreprise ou particulier) ou un bien, un produit appartenant au bénéficiaire.

7. C'est notamment le cas pour la santé, la formation, la culture, ..., pour des particuliers, le conseil, la professionnalisation, la communication, ..., pour des entreprises.

8. Par exemple l'achat de billets à un distributeur automatique dans le cas du transport urbain.

9. Ou d'une profession libérale. 


\section{3.- Arbitrages en temps réel, instabilité de la qualité et processus d'évaluation}

Le service effectif, réellement dispensé auprès du bénéficiaire présente toujours un écart avec le service générique conçu et projeté par l'entreprise. En effet, les salariés, en situation de face-à-face, sont constamment amenés à réaliser des arbitrages entre les orientations et objectifs fixés par la hiérarchie (de l'entreprise, de l'organisme public ou para-public), la spécificité de la demande du bénéficiaire incarnée par sa présence corporelle, sa subjectivité, et leur propre représentation de leur mission. De ce point de vue, les salariés sont amenés à prendre en charge les « évènements » (Zarifian, 1995) co-substantiels aux activités de service, à faire face aux situations dans leur hétérogénéité. De plus, à l'opposé des activités manufacturières où il est toujours possible de revenir sur l'output, améliorer sa qualité, rectifier ses attributs afin qu'ils correspondent aux normes, les services se déroulant en temps réel sans stocks intermédiaires ni finaux, ont une qualité qu'il n'est pas possible de rectifier. Il est possible, au besoin de compenser des qualités jugées insuffisantes, trop éloignées des attentes des bénéficiaires, mais les effets du service initial sur le bénéficiaire sont là, leur compensation engageant toujours, la réalisation d'un nouveau service, une nouvelle relation de face-à-face. Ces processus d'arbitrage conduisent, ainsi, à une qualité du service rendu, non homogène, d'un bénéficiaire à un autre, d'une organisation à une autre, d'un site à un autre, d'un moment à un autre. La qualité n'est donc pas stable.

Cette instabilité de la qualité de la prestation conduit les salariés à s'interroger constamment sur la notion de «travail bien fait », sur leur part de responsabilité quant à l'écart qui apparaît entre les attentes des bénéficiaires et leur perception des effets utiles du service rendu, sur les possibilités de faire évoluer la prestation. Cette interrogation entre en conflit ou en résonance avec l'interprétation de cette instabilité de la qualité qu'opère le management. Selon le fait que la hiérarchie de l'entreprise interprète l'écart entre le service générique (ce qu'elle a conçu) et le service effectif comme un dysfonctionnement ou comme un écart co-substantielle au type d'activité engagée, les salariés se trouvent déstabilisés ou au contraire reconnus et accompagnés. L'interprétation en termes de dysfonctionnement conduit à la culpabilisation des salariés considérés comme responsables de ne pas avoir suivi le prescrit de manière suffisamment précise ; l'interprétation en termes d'écart renvoie à une réflexion sur l'évaluation de la qualité, sur les ressources nécessaires à mobiliser pour faire face aux évènements, sur une possibilité de valoriser ces évènements par l'innovation.

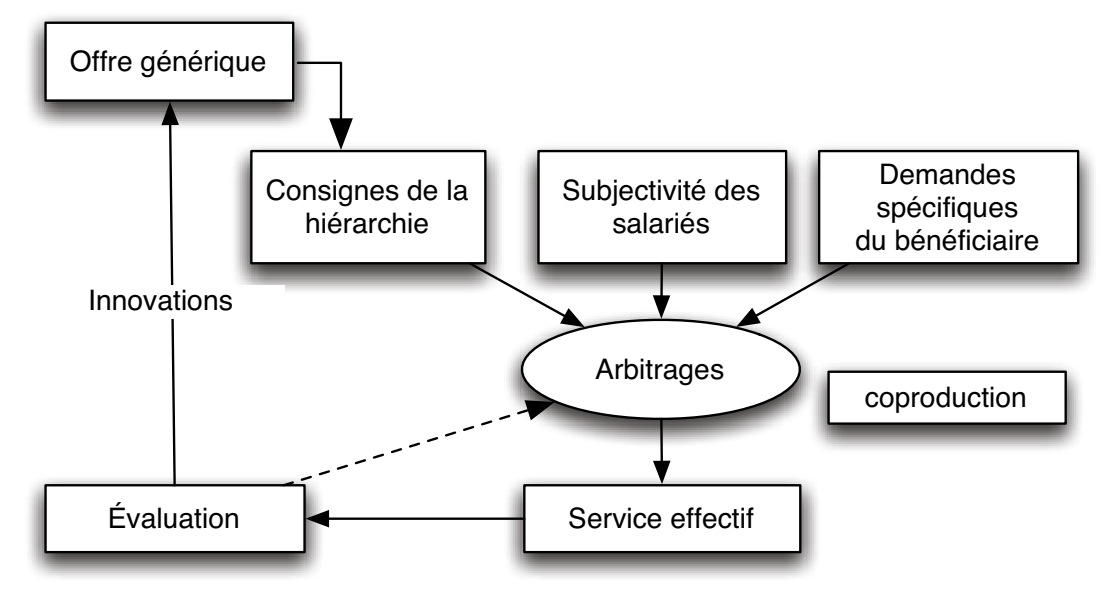

Figure 1.- Le processus d'arbitrage au coeur de la dynamique des services. 
Ces considérations sont d'autant plus importantes à prendre en compte que la réalisation des arbitrages (cf. du Tertre, \& Blandin, 2001 et figure 1) est engagée dans un processus dynamique qui intègre toujours des moments d'évaluation. Ces évaluations peuvent se limiter à de l'auto-évaluation, à des jugements de valeur entre pairs instruits de manière aléatoire, à des jugements de la hiérarchie fondés sur les seuls objectifs mesurables ; mais, ces évaluations peuvent être, aussi, organisées afin de dégager des construits partagés au sein d'un même collectif de travail, entre les collectifs de pairs et la hiérarchie, renvoyant au travail réel et aux ressources mobilisées (ou à mobiliser). Ces processus d'évaluation quelle que soit leur modalité de réalisation, font toujours retour sur de nouveaux arbitrages, sur des adaptations conscientes ou inconscientes, formalisées ou non-formalisées, voire sur des processus d'innovation incrémentale ou radicale. Les « services effectifs » sont, ainsi, l'expression d'un «construit social », reposant sur des processus d'évaluation plus ou moins explicites et formalisés, dont la légitimité fait débat au sein de la hiérarchie, des collectifs de salariés et des bénéficiaires. Ces débats concernent, notamment, l'intensité du travail.

\section{2.- L'impact de la « composition organique du temps de travail » sur la santé}

De manière traditionnelle, les économistes considèrent que l'intensité du travail est associée à une accélération des rythmes de travail ou à une extension des heures de travail. Cette intensification du travail se traduit par une augmentation de la valeur produite par tête. Elle provoque, généralement, une détérioration des conditions de travail qui peut s'exprimer par des contre-coûts humains lourds ${ }^{10}$, notamment en termes de santé. Cette approche « classique » suppose que deux préalables soient réunis : la qualité du produit doit être stabilisée et contrôlée ex-post de telle manière que l'intensification du travail n'ait pas d'incidence sur la qualité des produits présentés sur les marchés ; une coupure relativement nette doit être établie entre le temps de travail et le temps hors travail de telle manière que le contrôle du travail puisse s'opérer sur le lieu de travail.

Les activités immatérielles et relationnelles vont remettre en cause ces deux préalables, ce qui va avoir des conséquences sur les formes que va prendre l'intensité du travail et son impact sur la santé. Plusieurs observations méritent d'être formulées conduisant à renouveler les problématiques économiques abordant les liens intensité du travail et santé au travail. Elles concernent le «statut économique » du temps de travail, ce que nous dénommons « la composition organique du temps de travail », d'une part, les corrélations intensité du travail, qualité du service et santé, d'autre part.

\section{1.- La « composition organique du temps de travail » et l'intensité du travail}

Une question centrale se pose quant au rapport de l'entreprise au temps de travail : dans quelle mesure cette dernière tient-elle compte, dans son organisation et sa gestion, du caractère hétérogénéité de la finalité économique du temps de travail. De manière traditionnelle, le taylorisme et le fordisme avaient habitué les directions d'entreprise et les syndicats de salariés à opérer une distinction du contenu économique du temps de travail sur la base de catégories de salariés différentes. Pour illustrer ce propos, il est possible de considérer, dans un cadre industriel, que les O.S. assuraient essentiellement le travail de production directe ; les régleurs, les contrôleurs qualité, les outilleurs, les opérateurs chargés de la maintenance, ..., le travail de régulation; et les techniciens comme les

10. De ce point de vue, Marx (1971) a insisté sur la différence très nette à introduire entre les gains de productivité réalisés sur la base du progrès technique (ou d'une meilleur organisation du travail) et ceux réalisés par intensification du travail. Cependant les deux dimensions sont souvent liées, l'innovation technologique permettant d'accroître les rythmes ; c'est, notamment, le cas des processus de mécanisation au sein des chaînes fordiennes de montage. 
ingénieurs des bureaux des méthodes ou des services de conception, un travail relevant d'un investissement. En d'autres termes, les différents types de temps étaient gérés de manières différentes selon leur affectation à des catégories de salariés aux statuts différenciés. Dans les activités immatérielles et relationnelles, «les temps de production directe», «les temps de régulation » et «les temps consacrés aux investissements immatériels » ne sont généralement pas affectés à des catégories de salariés spécifiques. Les personnes ou les collectifs assurent toujours, quel que soit leur statut, une certaine combinaison de ces trois types de temps. Cette combinaison est dénommée « composition organique du temps de travail » (cf. du Tertre, 2001 et schéma 2).

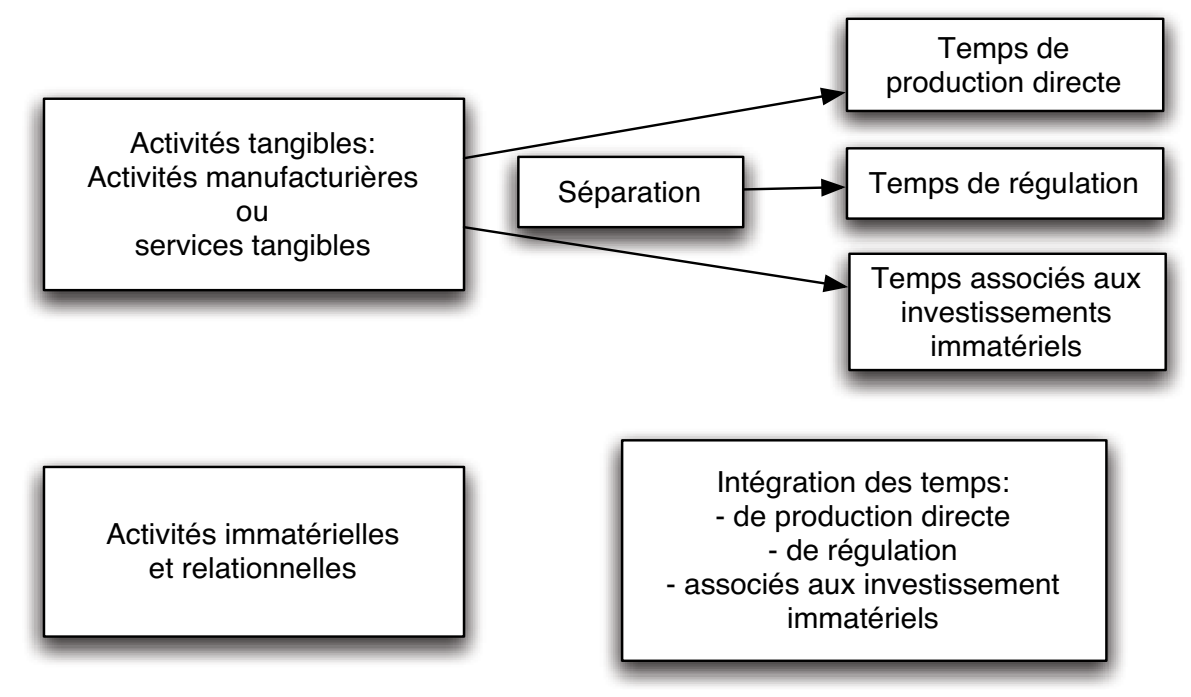

Figure 2.- La composition organique du temps de travail

Dans ce cadre d'analyse, l'identification et le mode de gestion des «temps apparemment connexes », c'est-à-dire des temps de régulation et des temps associés aux «investissements immatériels », ont une incidence sur les capacités des salariés à prendre en charge la production directe et les efforts que cela leur demande. Ces temps constituent des points d'appui, c'est-à-dire des ressources ou des manques lorsqu'ils ne sont pas suffisamment pris en compte, car la charge de travail est d'autant plus lourde à porter en situation de face-à-face que ces temps sont mal gérés.

Les dispositifs de régulation permettent de produire des références utiles à l'action, car élaborées collectivement sur la base du travail réel sous contraintes d'objectifs. Ces dispositifs sont des points d'appui pour faire la distinction entre des dysfonctionnements et des écarts dus aux évènements, ou identifier l'origine des dysfonctionnements. Lorsqu'ils mobilisent les cadres opérationnels, ils permettent à la hiérarchie d'être en prise avec la réalité du travail. Enfin, ces dispositifs peuvent faciliter l'introduction de tiers dans les situations potentiellement conflictuelles, ...

Les temps réservés aux investissements immatériels permettent, de leur côté, d'engager des réflexions sur la pertinence de l'organisation, sur les innovations à engager en termes d'outils de suivi et de gestion, de procédures d'évaluation. Ces temps destinés, également, à la professionnalisation par la formation, par les échanges d'expériences entre pairs, par l'appropriation de «l'état de l'art », par l'appropriation de connaissances scientifiques utiles à la compréhension de l'activité, renforcent les compétences des salariés et les aident à assumer leur mission en situation de face-àface.

En définitive, la dynamique des temps dits « connexes » permet de prendre en charge différemment le travail direct, en offrant des espaces d'élaboration cognitif individuel et collectif, des espaces d'élaboration psychique, dimension que suscitent, constamment, les cinq registres de l'intersubjecti- 
vité sollicités par la «relation de service». La qualité de «la composition organique du temps de travail » a donc une incidence sur les ressources dont dispose un salarié pour agir, sur ses marges de manœuvre face aux évènements, notamment ceux associés à la présence du bénéficiaire, à la diversité et la variabilité des situations de face-à-face. Elle a donc une incidence directe sur l'intensité du travail (à la hausse ou à la baisse), et conjointement sur la santé au travail dans la mesure où l'on considère que cette dernière est dépendante du lien qui se réalise entre la capacité d'élaboration psychique des salariés, d'un côté, leur capacité à penser et réaliser l'opération considérée comme opportune, de l'autre. Les construits sociaux à propos des « temps apparemment connexes » sont donc des enjeux essentiels pour «canaliser» l'intensité du travail et traiter de la santé au travail, même s'ils apparaissent souvent fragiles, constamment à bâtir et à rebâtir.

Cette incidence de la «composition organique du temps de travail»sur l'intensité du travail et la santé va avoir d'autant plus d'effet que le temps de travail a tendance à envahir la sphère de la vie privée. En effet, la coupure temps de travail / temps hors travail est de plus en plus difficile à établir de manière précise dans la mesure où la subjectivité des salariés est mobilisée (Boulin, \& du Tertre, 2001). Déjà, dans les activités industrielles, l'automatisation flexible avait inversé le rapport de l'homme à la machine-outil; l'homme étant amené à devancer les pannes et les dysfonctionnements ; à se créer sa propre représentation de l'activité et de sa régulation (du Tertre, \& Santilli, 1992). Dans les activités immatérielles et relationnelles, l'intersubjectivité associée à la « relation de service » mobilise la subjectivité du salarié encore plus radicalement.

Dans cette perspective, le travail d'élaboration, de résolution de problème, ..., se poursuit en dehors du site, en dehors des heures reconnues comme relevant du travail. On « emporte » avec soi son travail, ses difficultés relationnelles, on en rêve, on y pense, ... et ce d'autant plus que les processus de régulation sont mal assurés ou que les investissements immatériels sont peu pris en charge par l'entreprise. Cette évolution conduisant à la porosité du temps de travail et du temps hors travail est favorisée par la diffusion des NTIC ${ }^{11}$, notamment des téléphones portables et des ordinateurs portables $^{12}$. Les construits sociaux autour de la qualité «de la composition organique du temps de travail » exigent, ainsi, la prise en compte de ses effets dans la sphère privée, hors travail.

\section{2.- Arbitrage : intensification du travail / qualité du service / santé}

Lorsque les construits sociaux sont défaillants à propos de « la composition organique du temps de travail », la croissance des objectifs assignés au temps de travail direct va provoquer une intensification du travail relevant de deux phénomènes s'auto-renforçant. D'un côté, le management et les salariés, eux-mêmes, vont chercher à étendre le temps de travail direct au détriment des temps de régulation et des temps assignés aux investissements immatériels, en considérant qu'il est nécessaire d'aller à « l'essentiel ${ }^{13}$. En privilégiant, ainsi, les situations de face-à-face au détriment des temps de régulation et des temps dédiés aux investissements immatériels, ce processus affaiblit les ressources dont disposent les salariés pour assumer la réalisation des nouveaux objectifs. D'un autre côté, la

11. Inversement, la vie personnelle de chacun est introduite, également, dans le site de travail ; les enjeux personnels venant s'articuler à la disponibilité psychique des salariés. La qualité de la vie hors travail vient, ici, s'articuler avec celle de la vie au travail.

12. Cet entrelacement entre la sphère du travail et la sphère du privé est l'une des difficultés qu'ont rencontrées les entreprises et les partenaires sociaux pour discuter et élaborer des accords sur la réduction du temps de travail. En fait, des construits très différents de « composition organique du temps de travail » se sont mis en place au sein d'une même branche ou au sein d'une même entreprise, alors qu'un même référent « trente-cinq heures » était affiché. Une question centrale émerge : le mode d'émergence (de légitimité, de stabilité) d'un construit concernant cette « composition organique » et ses effets sur le lien temps de travail sur site / temps de travail hors site.

13. C'est une réaction que l'on a pu observée dans de nombreuses situations dans le cadre de l'application de la loi sur les trente-cinq heures. 
pression qui s'opère sur les temps connexes affaiblit les capacités d'innovation de l'entreprise concernant leur usage, au moment même où leur contenu devrait être repensé et adapté aux nouveaux objectifs. La conjugaison de ces deux phénomènes provoque un accroissement de la charge et de la pression qui s'exercent sur les salariés ${ }^{14}$. Les risques de détérioration de la santé au travail sont, alors, majeurs.

Dans de telle situation d'intensification du travail direct, les salariés ont deux types de réactions possibles : l'une consiste à prendre sur soi avec un « déversement» des activités de « régulation » et des « investissements immatériels » vers la vie privée, au détriment de cette vie privée ; l'autre consiste à engager une stratégie défensive de renfermement sur soi dans le travail et de mise à distance des bénéficiaires au détriment de la qualité du service.

La première attitude n'a pas d'effet immédiat sur la qualité du service rendu, dans la mesure où le salarié s'appuie sur ses ressources subjectives pour faire face aux nouveaux objectifs d'ordre quantitatif, et répondre aux attentes qu'il perçoit chez le bénéficiaire, en termes de qualité de service. Il tente dans un premier temps de réaliser les régulations nécessaires à la qualité du service dans des temps hors travail, à assumer par des réseaux extérieurs à son travail des formes d'investissements immatériels, notamment dans le domaine des connaissances. Mais cette stratégie se heurte à deux limites :

- la première concerne le manque d'efficacité des régulations non pilotées par l'organisation et les difficultés de les capitaliser au sein du collectifs de travail. L'ampleur de l'énergie déployée ne trouve pas de contre partie en termes de reconnaissance. Cette voie conduit à des phases d'épuisement, exprimées souvent par des périodes dépressives portant atteinte à la santé ;

- la seconde concerne les perturbations que provoquent cette excroissance du temps de travail sur la vie familiale, la vie de couple et la vie sociale ; ce qui ne reste pas sans effet sur la santé du salarié ou de son entourage.

La seconde attitude consiste à développer des stratégies défensives. Lorsque les contraintes de temps se tendent, certains salariés sont amenés à développer des comportements leur permettant de limiter l'engagement subjectif qu'appelle toute « relation de service ». Ils mettent en œuvre des attitudes « écran », des « comportements réflexes » pour s'extraire de la pression. Ces comportements peuvent s'exprimer sous forme de tics de langage, de «tics d'attitude », de «tics de posture », de rejets «systématiques » de l'autre, ... L'ensemble de ces attitudes ont, de fait, une fonction de protection du salarié en limitant son engagement donc sa vulnérabilité.

Ces attitudes de repli sont possibles dans la mesure où toutes ces activités immatérielles et relationnelles mettent en œuvre des processus d'arbitrage en temps réel, comme nous l'avons analysé précédemment, conduisant à des construits instables. Un renforcement de l'intensité du travail recherché par la hiérarchie, considéré comme non légitime, se traduit, ici, par une baisse de la qualité du service rendu. Dans cette perspective, il faut noter que le renforcement de l'intensité du travail est partiellement fictive, la baisse de la qualité du service effectif conduisant à un service non réalisé ${ }^{15}$. Mais, attention, l'arbitrage opéré par le salarié entre les objectifs d'intensification du travail et la qualité du service rendu, n'est pas sans effet sur sa santé. Car, les salariés vivent, généralement, mal cette baisse de la qualité réelle du service rendu provoquant la dégradation du regard des bénéficiaires sur eux (Dejours, 2000).

14. Les interrogations sociales actuelles sur l'intensification du travail et ses impacts sur la santé sont d'autant plus urgentes à mener que de nombreuses entreprises de services, ou de nombreux organismes publics et para-publics (dans le domaine de la santé, notamment) avaient engagées des processus de réduction de temps de travail sans prise en compte de l'importance de la qualité de leur « composition organique du temps de travail ».

15. La partie non rendue du service, pour cause de trop forte intensité du travail exigée, conduit, sur un plan économique, à une dynamique récessive ; le service réel ne correspondant que partiellement au service générique (affiché). 
En définitive, soit le salarié répond aux injonctions de la hiérarchie et/ou aux attentes des bénéficiaires avec un risque «d'envahissement» et d'épuisement qui atteignent, in fine, le psychique et le corps; soit le salarié met à distance les attentes des «partenaires » (hiérarchie / bénéficiaires). Il prend, alors, le risque d'un affaiblissement de la reconnaissance de l'autre et d'une atteinte à l'image de soi qui ne restent pas sans effet sur sa propre santé. Dans ce dernier cas de figure, ces formes de « protection » se retournent d'un côté, contre les salariés et leur santé, dans les dimensions physiologiques et psychologiques, de l'autre, contre les bénéficiaires à travers l'inadéquation de la prestation vis-à-vis de leurs attentes ou de leurs besoins ${ }^{16}$.

\section{3.- Construits et dispositifs institutionnels}

L'ensemble des phénomènes analysés ci-dessus constituent le cadre dans lequel se repose, sur un plan économique et analytique, la question de l'intensité du travail et de son rapport à la santé. L'enjeu des construits sociaux et des dispositifs institutionnels qui les appuient, est au cœur d'une réflexion pluridisciplinaire nécessaire.

\section{1.- L'enjeu transversal de la notion de « construits »}

Pour reprendre de manière synthétique la problématique développée, quatre dimensions du travail ont une incidence sur son intensité et doivent retenir notre attention :

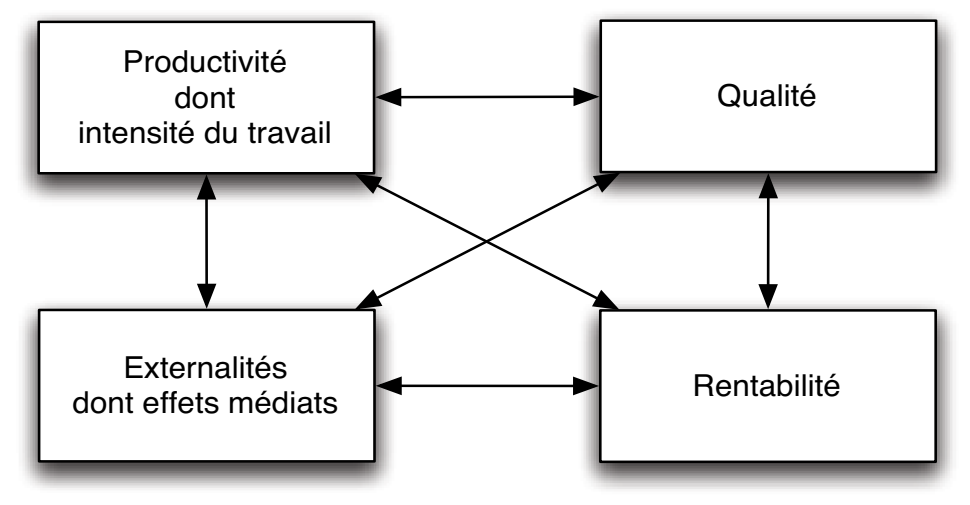

Figure 3.- Les quatres registres de la performance

- la subjectivité et l'intersubjectivité mobilisées dans le travail conduisent à rendre la frontière temps de travail / temps hors travail difficile à identifier et à stabiliser, les NTIC venant renforcer le phénomène. Ce constat conduit à se préoccuper des liens entre la santé au travail et la santé hors travail, les conditions psychiques et physiologiques de l'activité de travail étant le point nodal de l'articulation. D'un point de vue économique, cela signifie que la question des externalités négatives des organisations du travail doit être considérée dans une approche renouvelée de la performance ${ }^{17}$ des entreprises (figure 3 ) ;

- le statut économique du temps de travail se complexifie, la qualité de la « composition organique du temps de travail » ayant un impact direct sur l'intensité du travail. L'approche en termes de «configuration organique du temps de travail» conduit à souligner les effets des «temps connexes » tant sur la performance de l'entreprise que sur la santé au travail ;

16. La violence au travail dans les situations de face-à-face, produite ou subie, est une forme de résultante de ces processus individuels et socio-économiques. 
- l'irruption du bénéficiaire au sein du processus de travail, de son organisation, de son pilotage, ..., conduit à des arbitrages non stabilisés qui renouvellent constamment la qualité du service rendu, les régulations opératoires à trouver et à mettre en œuvre, l'intensité du travail assurée par le prestataire ;

- les tensions entre les différents registres de la performance (schéma 3) apparaissent suivant des corrélations qui ne se limitent pas au duo intensité / rentabilité mais qui engagent aussi la qualité et les externalités (du Tertre, \& Blandin, 2001).

Pour chacun de ces aspects, un « construit social » existe ; il est d'autant plus légitime aux yeux des acteurs que son élaboration s'appuie sur un dispositif institutionnel reconnu par les acteurs comme en phase avec la réalité du travail et des arbitrages à opérer. Légitimité de nouveaux construits et émergence de nouveaux dispositifs institutionnels adaptés aux caractéristiques des activité immatérielles et relationnelles sont, ainsi, intrinsèquement liés. Ce sont des questions clés de la réflexion et de l'action. Cela concerne autant :

- la définition de règles quant au rapport entre le travail sur site et le travail hors site, notamment à propos de l'usage des outils transportables de traitement de l'information ;

- l'évaluation de la qualité des effets des services et de l'engagement de chaque acteur dans sa production ;

- la conception de nouveaux outils de gestion permettant de prendre en compte la « composition organique du temps de travail » et notamment les investissements immatériels ;

- l'émergence de processus d'évaluation permettant aux acteurs de se construire un point de vue, intégrant les dimensions de la santé au travail.

Il s'agit de renvoyer l'exercice de la production de valeur aux conditions d'exercice réel du travail. Il s'agit donc de faire du travail un axe central de réflexion, d'évaluation afin qu'un lien s'opère entre le développement des ressources ${ }^{18}$ que mobilise et affecte le travail, et le développement des richesses que procure le travail.

En définitive, les processus d'évaluation doivent être en mesure non seulement d'établir un jugement sur les résultats de l'activité, mais également sur le processus engagé pour les obtenir, c'est-àdire sur le travail réel, les ressources dont il a besoin pour opérer et la façon dont ces ressources sont affectées (ou développées) par l'activité. Les procédures d'évaluation représentent, alors un travail sur le travail devant permettre d'instruire la pertinence et la légitimité des construits.

\section{2.- Renouveler l'approche de la pensée à l'action}

Dans cette perspective, la théorie économique hétérodoxe et, notamment, la théorie de la régulation comme la théorie des conventions sont interpellées sur deux aspects essentiels :

- une capacité à lier subjectivité, intersubjectivité et dynamique de création de valeur ;

17. Ces tensions se compliquent car elle mettent en jeu, au delà du couple intensité du travail / qualité, deux autres registres de la performance. En effet, la performance d'une entreprise ou d'un organisme ne se limite pas au registre de la productivité et de la qualité. S'y adossent des enjeux d'externalités positives ou négatives (c'est-à-dire des effets externes aux prestataires et bénéficiaires directs), des enjeux de rentabilité financière ou d'équilibre budgétaire.

De ce point de vue, les quatre registres de la performance ne sont pas indépendants les uns des autres. Mais leurs corrélations, notamment, au sein des activités immatérielles et relationnelles sont difficilement analysables et évaluables. Cela tient aux outils de gestion qui ne sont pas aujourd'hui en phase avec ces enjeux. Ils sont encore très fortement marqués par les approches tayloriennes voire fordiennes du travail associées aux dynamiques industrielles (du Tertre, \& Blandin, 2001).

18. Certains économistes considèrent que cette approche est en résonance avec la notion de développement durable 
- une capacité à lier subjectivité, dispositifs institutionnels et théorie de l'action pour sortir de la tendance normative de la théorie économique.

Dans ce cadre des liens doivent se construire avec les approches gestionnaires pour permettre l'élaboration de nouveaux modèles de gestion. En effet, les modèles de gestion actuels, dominants dans les entreprises ou les organismes publics créent de fortes tensions avec les processus analysés dans cet article. Ces tensions tiennent aux difficultés des approches gestionnaires :

- à sortir de modèles qui privilégient toujours le registre de la rentabilité vis-à-vis des autres registres de la performance ;

- à élaborer des outils d'évaluation qui articulent résultats et processus ;

- à rendre compte de la portée économique hétérogène du temps de travail, notamment à intégrer les investissements immatériels.

Si différents «modes de régulation » des liens intensité du travail - santé sont apparaissent possibles, encore faut-il que les outils de gestion évoluent dans leur rapport au travail réel ; car ces outils ont un impact puissant sur les modes de décision au sein des organisations. Une pensée pluridisciplinaire à propos du travail sera d'autant plus pertinente qu'elle trouvera son mode d'accès aux enjeux gestionnaires. Elle sera, alors, convoquée comme un moment de l'action.

\section{4.- Conclusion}

Sur le plan théorique, l'intensité du travail interroge l'économiste quant aux liens qui s'établissent entre subjectivité et travail en tant qu'activité, entre subjectivité mobilisée par l'activité réelle et « construits sociaux », entre subjectivité et dispositifs institutionnels. L'approche de l'intensité du travail peut alors être renouvelée dans une perspective qui intègre les nouveaux enjeux associés aux caractéristiques des activités immatérielles et relationnelles.

Sur le plan de l'outillage permettant d'appréhender l'intensité du travail, il apparaît nécessaire qu'une dynamique pluridisciplinaire opère afin de prendre la mesure de l'ensemble des champs concernés par ces enjeux. Les cinq registres de l'intersubjectivité associée aux « relations de service » qui sont au cœur des activités immatérielles, invitent à la pluridisciplinarité pour analyser de manière précise les liens qui s'établissent entre l'intersubjectivité, les systèmes de défense ou d'évitement et les effets sur la santé, notamment en termes de TMS. La compréhension des conditions d'émergence des « construits » exige, en effet, une forte coopération entre les disciplines du travail. Sont, tout particulièrment, sollicités, à côté de l'économie et de la gestion, la psychologie du travail, la sociologie, le droit et l'ergonomie.

\section{BIBLIOGRAPHIE}

Boulin, J.-Y,. \& Tertre du C. (2001). L'impact de la réduction-aménagement du temps de travail sur les usages du temps. Rapport de recherche pour le Secrétariat au tourisme et pour le Commissériat Général du Plan, Document IRIS, Paris.

Boyer, R. (1990). Les problèmes de la régulation face aux spécificités sectorielles. Cahiers d'Économie et de Sociologie Rurales, $\mathrm{n}^{\circ} 17$.

Boyer, R., \& Saillard, Y. (2002). La théorie de la régulation, l'état des savoirs. Paris: Édition La Découverte (deuxième édition augmentée).

Dejours, C. (2000). Travail, usure mentale. Paris: Bayard (nouvelle édition augmentée).

Gadrey, J. (1992). L'économie des services. Paris: Édition La Découverte, collection Repères.

Gadrey, J. (1996). Services : la productivité en question. Paris: Desclée de Brouwer, collection sociologie économique. 
Marx K. (1971). Les chapitres inédits du capital. Paris: Seuil, collection 10-18.

Tertre du, C. (1989). Technologie, flexibilité, emploi : une approche sectorielle du post-fordisme. Paris: L'Harmattan.

Tertre du, C. (2001). Nouveau régime d'accumulation et Aménagement-Réduction du temps de travail : le rôle 'clé' des activités immatérielles et relationnelles. In G. Groux (Ed.), L'action publique négociée : approche à partir des 35 heures (France - Europe), (pp. 219-233). Paris: l'Harmattan.

Tertre du, C. (2002). Une approche sectorielle du travail. In R. Boyer, \& Y. Saillard (Eds), La théorie de la régulation, l'état des savoirs, (pp. 323-331). Paris: La Découverte (deuxième édition augmentée).

Tertre du, C., \& Blandin, O. (2001). Performance des activités de service : le cas de La Poste en zone urbaine sensible. Paris : $\mathrm{n}^{\circ} 9$ de la collection de la mission Recherche de La Poste.

Tertre du C., \& Santilli, G. (1992). Automatisation et travail. Paris: PUF, collection économie en liberté.

Zarifian, P. (1995). Le travail et l'événement. Paris: L'Harmattan.

\section{RÉFÉRENCEMENT}

Tertre du, C. (2005). Services immatériels et relationnels : intensité du travail et santé. @ ctivités, 2 (1), 37-49, http://www.activites.org/v2n1/dutertre.pdf

\section{RESUMEN}

El lugar que ocupan los servicios inmateriales y aquellos que implican una relación en la dinámica económica y en el crecimiento es cada vez más importante. Por esta razón, las empresas y los organismos públicos intentan racionalizarlos.

En base a una caracterización de la economía inmaterial, el artículo destaca la importancia de los tiempos de regulación y de los tiempos destinados a las inversiones inmateriales, sobre los modos de regulación de la intensidad del trabajo y de su impacto sobre la salud.

\section{PALABRAS CLAVE}

Servicios, relación de servicio, inmaterial, intenidad del trabajo, salud.

\section{RÉSUMÉ}

Les services immatériels et relationnels occupent une place de plus en plus importante dans la dynamique économique et la croissance. C'est pourquoi les entreprises et les organismes publics tentent de les rationaliser. Sur la base d'une caractérisation de l'économie immatérielle, l'article met en évidence l'importance des temps de régulation et des temps destinés aux investissements immatériels sur les modes de régulation de l'intensité du travail et de son impact sur la santé.

\section{MOTS CLÉ}

Services, relation de service, immatériel, intensité du travail, santé. 